\title{
ApoSense: a novel technology for functional molecular imaging of cell death in models of acute renal tubular necrosis
}

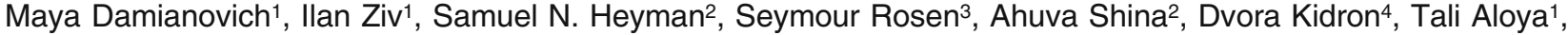

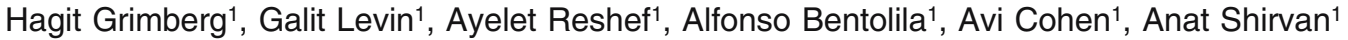 \\ ${ }^{1}$ NeuroSurvival Technologies (NST) Ltd., 5 Odem St., Kiryat Matalon, Petah Tikva, 49170, Israel \\ 2 Department of Medicine, Hadassah Hospital, Mt.Scopus and the Hebrew University Medical School, Jerusalem, Israel \\ ${ }^{3}$ Department of Pathology, Beth Israel Deaconess Medical Center and Harvard Medical School, Boston, MA, USA \\ ${ }^{4}$ Department of Pathology, Meir Hospital, Kfar-Saba, Israel
}

Received: 15 March 2005 / Accepted: 4 July 2005 / Published online: 29 November 2005

(C) Springer-Verlag 2005

\begin{abstract}
Purpose: Acute renal tubular necrosis (ATN), a common cause of acute renal failure, is a dynamic, rapidly evolving clinical condition associated with apoptotic and necrotic tubular cell death. Its early identification is critical, but current detection methods relying upon clinical assessment, such as kidney biopsy and functional assays, are insufficient. We have developed a family of small molecule compounds, ApoSense, that is capable, upon systemic administration, of selectively targeting and accumulating within apoptotic/necrotic cells and is suitable for attachment of different markers for clinical imaging. The purpose of this study was to test the applicability of these molecules as a diagnostic imaging agent for the detection of renal tubular cell injury following renal ischemia.

Methods: Using both fluorescent and radiolabeled derivatives of one of the ApoSense compounds, didansyl cystine, we evaluated cell death in three experimental, clinically relevant animal models of ATN: renal ischemia/reperfusion, radiocontrast-induced distal tubular necrosis, and cecal ligature and perforation-induced sepsis.

Results: ApoSense showed high sensitivity and specificity in targeting injured renal tubular epithelial cells in vivo in all three models used. Uptake of ApoSense in the ischemic kidney was higher than in the non-ischemic one, and the
\end{abstract}

M. Damianovich and I. Ziv had an equal contribution to the paper. Potential conflict of interest: ApoSense is a product developed by NST, Ltd. NST financially supported the research presented in full. M. Damianovich, I. Ziv, T. Aloya, H. Grimberg, G. Levin, A. Reshef, A. Bentolila, A. Cohen, and A. Shirvan are NST employees. S.N. Heyman, S. Rosen , A. Shina, and D. Kidron have no actual or potential conflict of interest in relation to this article. ApoSense is still an investigational product.

Ilan Ziv (•)

NeuroSurvival Technologies (NST) Ltd.,

5 Odem St., Kiryat Matalon,

Petah Tikva, 49170, Israel

e-mail: Ilan@nst.co.il

Tel.: +972-3-9215717, Fax: +972-3-9227581 specificity of ApoSense targeting was demonstrated by its localization to regions of apoptotic/necrotic cell death, detected morphologically and by TUNEL staining.

Conclusion: ApoSense technology should have significant clinical utility for real-time, noninvasive detection of renal parenchymal damage of various types and evaluation of its distribution and magnitude; it may facilitate the assessment of efficacy of therapeutic interventions in a broad spectrum of disease states.

Keywords: Apoptosis imaging - Cell death detection Renal disease - Renal uptake - Small animal imaging

Eur J Nucl Med Mol Imaging (2006) 33:281-291

DOI 10.1007/s00259-005-1905-x

\section{Introduction}

Acute renal failure (ARF) that results from ischemic or toxic insults to the kidney is usually referred to clinically as acute tubular necrosis (ATN), where tubular damage and altered glomerular hemodynamics may coexist or even lead to each other [1-3]. In some cases of either human ARF [4, 5] or experimental models of renal injury [6], a lack of correlation between histological evidence of injury and renal function has been found, and the relative contribution of functional versus structural changes to the evolving renal dysfunction has not been unequivocally substantiated. Since ATN is a dynamic clinical condition, rapidly evolving from the incipient to the established phase, its early identification is critical. However, the various currently available methods of clinical and laboratory assessment are insufficient to differentiate between ischemic ARF, resulting in acute tubular dysfunction secondary to cell injury, and prerenal azotemia, characterized by decreased glomerular filtration but no epithelial cell injury. The inability to distinguish between these situations leads to long dis- 
putes over the pathogenesis, diagnosis, and management of evolving renal failure. Renal biopsy provides limited data because of the small sample size and the very focal pattern of tubular injury $[2,7]$. In addition and most importantly, it is impractical in the human syndrome. Functional data in early phases are also of little help, because prerenal azotemia becomes an important consideration at that time, and only the evolution of the clinical situation really defines the nature of the problem $[8,9]$. The utilization of specific urinary biomarkers of tubular injury, such as KIM1 , is not yet considered valid or sufficiently sensitive to establish ATN $[10,11]$. Moreover, these tests remain qualitative rather than quantitative markers of injury. Since therapeutic interventions might differ at various points in the evolution of ATN, advanced techniques are required to noninvasively follow the dynamic status of tubular injury. Furthermore, a technology that enables imaging endpoints, instead of time-consuming dissection and detection of renal parenchymal cellular damage, would be highly valuable.

Multiple lines of evidence suggest that both apoptotic and necrotic cell death are important pathogenic mechanisms in acute renal failure $[12,13]$, where renal parenchymal cells are the primary target of a broad spectrum of inciting factors, ranging from ischemic and nephrotoxic agents to endotoxemia. Apoptotic cell death is a feature of both ischemia-reflow injury [14] and radiocontrast-induced acute renal failure [15], and is a documented event in transplant biopsy as well [16]. The role of apoptosis in sepsis syndrome has not been adequately explored, but there is rapidly developing evidence to suggest that increased apoptotic processes may play a determining role in the outcome of sepsis syndrome $[17,18]$. Although apoptosis is recognized as playing a role in these conditions, there is a lack of sensitive markers to monitor programmed cell death in association with disease progression or regression.

Recently, we developed a family of small molecule compounds, ApoSense, capable of discriminating between vital and apoptotic cells. ApoSense molecules are small non-peptidic fluorescent compounds that are capable of selective targeting, binding, uptake, and accumulation within apoptotic and/or necrotic cell cytoplasm, from the early stages of the death process. Our preliminary results from different animal models associated with apoptosis showed that the recognition of damaged cells by ApoSense is universal, irrespective of the cell type or the apoptotic/ necrotic trigger. Indeed, examined in multiple animal models, ApoSense was proven to be a highly sensitive and specific sensor for in vivo detection of neoplastic, hepatic, myocardial, atherosclerotic, and neuronal apoptotic and necrotic cell death (in preparation). In the present study the potential of both fluorescent and radiolabeled derivatives of one of the ApoSense compounds, didansyl cystine (DDC), was explored as a diagnostic imaging agent for detection of renal tubular injury in three different in vivo animal models of ATN: renal warm ischemia/reperfusion, radiocontrast-induced distal tubular necrosis, and cecal ligature/perforation-induced sepsis. These different models illustrate the applicability of ApoSense as a diagnostic imaging agent enabling real-time accurate detection of renal parenchymal damage of various types, and evaluation of its distribution and magnitude.

\section{Materials and methods}

Male Sprague-Dawley rats weighing 180-250 g and 8- to 10-weekold C57Bl mice (Harlan Laboratories, Jerusalem, Israel) were used for these experiments. All experimental protocols were performed according to the Guiding Principles for Research Involving Animals, and approved by the local Animal Care Committee; the experiments described in this manuscript also comply with the current laws of Israel.

\section{ApoSense synthesis and radiolabeling}

The ApoSense compound used in the present study was $N, N^{\prime}$ didansyl-L-cystine (DDC). Both its inherent fluorescent characteristics and its radiolabeled derivative (labeled with ${ }^{3} \mathrm{H}$ radionuclide) were used for identification and quantification of apoptotic cells.

For DDC synthesis, dansyl chloride $(675 \mathrm{mg})$ and cystine (240 mg) were dissolved in water/acetone solution with potassium carbonate $(550 \mathrm{mg})$, and after $1.5 \mathrm{~h}$ at room temperature, the aqueous mixture was extracted with ethyl acetate. The crude DDC was dissolved in 5\% sodium carbonate, washed with ether, and reacidified to $\mathrm{pH} 3$ to afford a yellow precipitate. The product was analyzed using ${ }^{1} \mathrm{H}-\mathrm{NMR}$.

Labeling of DDC with ${ }^{3} \mathrm{H}$ was performed by consecutive addition of $0.16 \mathrm{mg}$ cystine $(0.65 \mu \mathrm{mol}), 0.57 \mathrm{mg}$ sodium carbonate $(5.3 \mu \mathrm{mol})$, and $37 \mathrm{MBq}(1 \mathrm{mCi})$ of ${ }^{3} \mathrm{H}$-dansyl chloride (Vitrax) at a concentration of $1.4 \times 10^{-4} \mathrm{mmol}$. The product was purified using a Bond-Elut C-18 cartridge eluted with $25 \%$ acetonitrile in $0.1 \%$ TFA in water, then lyophilized and dissolved in $1 \mathrm{ml}$ of NaPPi buffer $(0.1 \mathrm{M}, \mathrm{pH}=7.4)$. Radiochemical purity was assessed by thin-layer chromatography.

\section{Evaluation of ApoSense binding to apoptotic cells in vitro}

Human adult T-cell leukemia Jurkat cells (clone E6-1) and cervical carcinoma HeLa cells (CCL-2.2), were purchased from ATCC (Rockville, MD, USA) and grown in Dulbecco's modified Eagle's medium with $10 \%$ fetal calf serum at $37^{\circ} \mathrm{C}$ in $5 \% \mathrm{CO}_{2}$. For induction of apoptosis in the Jurkat cell sample, $1 \times 10^{6}$ cells $/ \mathrm{ml}$ were treated with IgM anti-Fas (CD95) antibody, clone CH11 (Medical and Biological laboratories, Japan) at a concentration of $0.1 \mu \mathrm{g} / \mathrm{ml}$ for 120-180 min. In additional studies, before incubation with anti-Fas antibody, Jurkat cells were subjected to $50 \mu \mathrm{M}$ of caspase inhibitor ZVAD-FMK in $0.05 \%$ DMSO (Enzyme System Products, Dublin, CA). For apoptosis induction in HeLa cells, growing cells were treated with staurosporin (STS; $250 \mathrm{n} M$; Sigma) for $18 \mathrm{~h}$. At the end of the incubation period, cells were harvested by trypsinization (Trypsin-EDTA, Bet-Haemek, Israel) and collected by centrifugation.

For assessment of apoptosis, both fluorescence microscopy and flow cytometry were employed. Trypsinized HeLa cells were incubated with DDC (50 $\mu M$ ) and annexin V-FITC (Phosphatidyl Serine Detection Kit, IQP-116F) for $20 \mathrm{~min}$ and the staining pattern was evaluated under a fluorescence microscope (BX51TF; Olympus Optical.Co., Ltd., UK) equipped with relevant filters. For DDC staining, excitation at 365 and emission at 420 (band pass) nm was employed, whereas for FITC detection, excitation was at $488 \mathrm{~nm}$ and emission at $530 \mathrm{~nm}$. Apoptotic Jurkat cells were detected by flow cytometry after double staining with annexin V-FITC and PI or with 


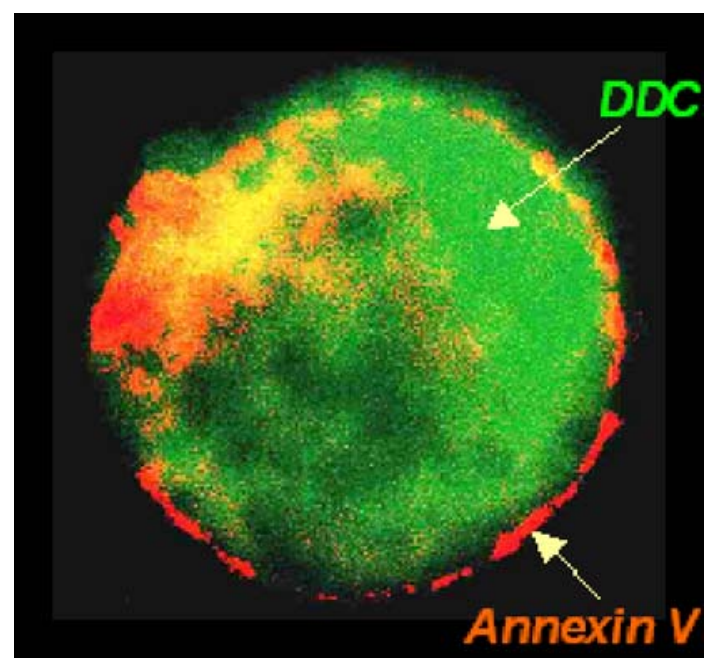

Fig. 1. Concomitant confocal imaging of HeLa cell undergoing apoptosis by DDC (green fluorescence) and annexin V (red fluorescence). DDC accumulates within the cytoplasm of the apoptotic cell while annexin $\mathrm{V}$ is attached to the external membrane

DDC and PI. Briefly, $30 \mu \mathrm{l}$ of cell suspension $\left(1 \times 10^{6}\right.$ cells $\left./ \mathrm{ml}\right)$ was diluted in $300 \mu \mathrm{l}$ Hepes buffer $(10 \mathrm{~m} M$ Hepes and $140 \mathrm{~m} M \mathrm{NaCl}$, $\mathrm{pH}$ 7.4) containing annexin V-FITC/PI or $50 \mu M$ of DDC/PI. Following $40 \mathrm{~min}$ of incubation at room temperature, the cells were subjected to analysis using FACS Vantage VE (BD Biosciences, San Jose, CA) and CellQuest software. A total of 10,000 events were collected for each sample.

\section{Evaluation of ApoSense uptake into apoptotic/necrotic cells in vivo}

The detection of renal parenchymal cell death by ApoSense was assessed in three animal models of ATN, generally classified under ischemic and septic etiologies [19-21].

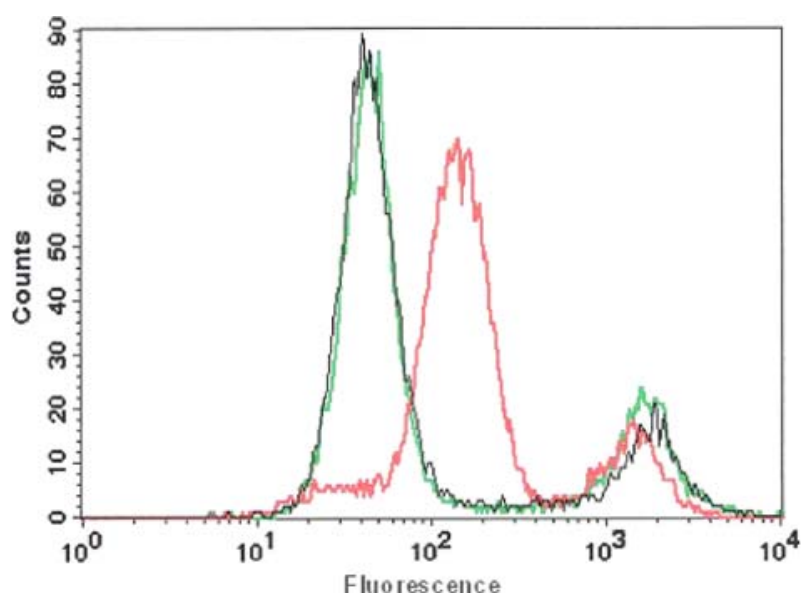

Fig. 2. Binding of DDC to apoptotic cells is caspase dependent. Jurkat cells were treated with CD95 in the absence (red line) or presence (black line) of the pan caspase inhibitor z-VAD-fmk for $150 \mathrm{~min}$. Following incubation, cells were centrifuged and incubated in the presence of DDC for $40 \mathrm{~min}$ before FACS analysis. This histogram charts the number of cells counted (y-axis) and the fluorescence intensity (x-axis). The green line represents the control non-apoptotic sample

Rat renal ischemia-reperfusion (I/R) model Operative procedures were performed in rats under general anesthesia induced by the combination of ketamine, $80 \mathrm{mg} / \mathrm{kg}$, and xylazine, $10 \mathrm{mg} / \mathrm{kg}$,
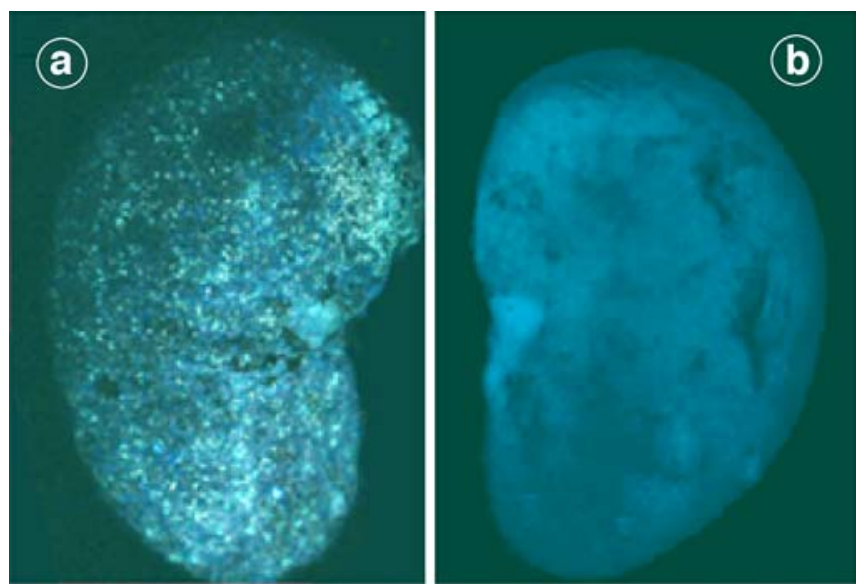
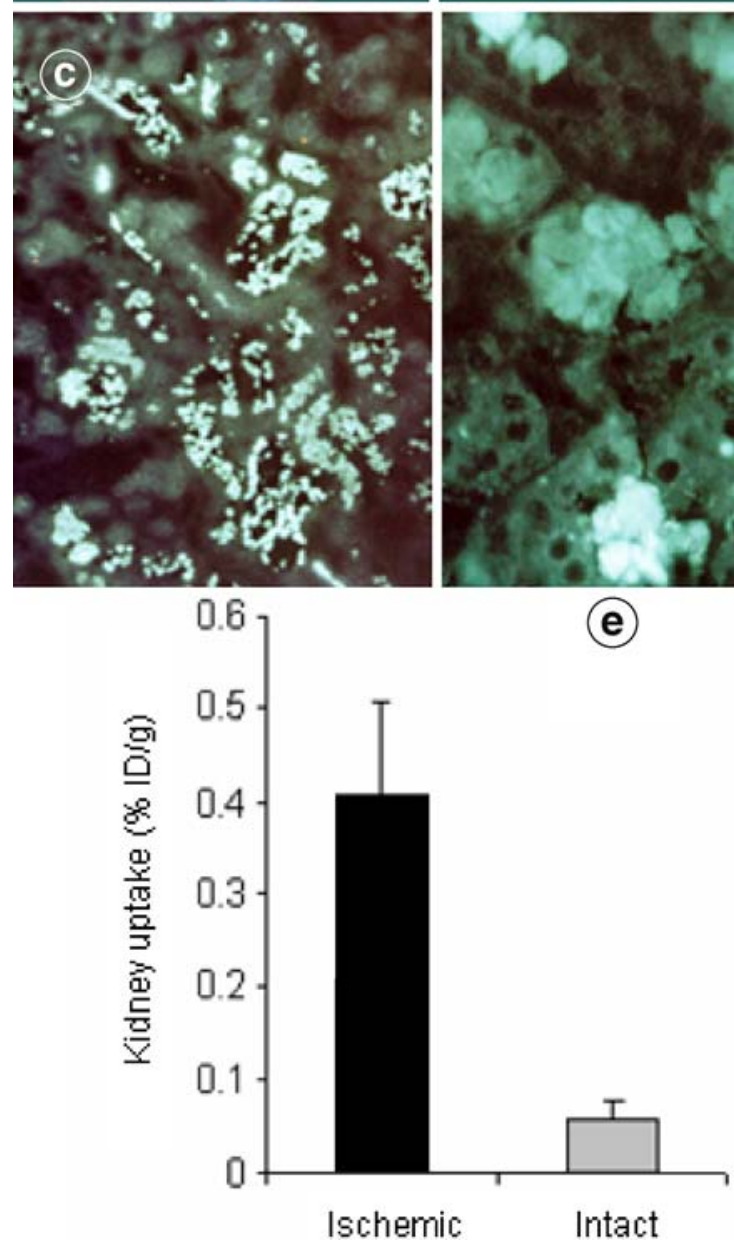

Fig. 3. DDC uptake into renal damaged areas. a,b Ex vivo images of the left ischemic (a) and the right sham-operated (b) kidney derived from a single $I / R$ rat $(\times 7)$. Whole organs were viewed under a stereomicroscope using a NU filter set without light flux, $4 \mathrm{~h}$ after DDC administration. c,d Histological sections of kidneys $(\mathbf{c}, \times 100$; d, $\times 600$ ) revealing intense uptake along the tubule epithelium or in the tubule lumen. e A quantitative estimate of DDC uptake into I/R ischemic and intact (sham) kidney from five rats, normalized to total injection dose for each gram of tissue. Values are means \pm s.e.m. 
Fig. 4. Morphological evidence for renal tubular cell apoptosis/ necrosis following ischemia-reflow. a Tubular cells $(\mathrm{H} \& \mathrm{E}$ staining) are seen with changes typical of apoptosis (left). In an adjacent $5-\mu \mathrm{m}$ section (right), these cells take up DDC. $\times 1,000$. b Necrotic tubular cells in tubular lumina (H\&E staining; left) and corresponding DDC fluorescence of the same injured cells (right). $\times 400$. c Co-localization of DCC fluorescence (right) with TUNEL-positive nuclear staining (left). Some cells are likely in the early stages of apoptosis and are TUNEL negative, but take up DDC (arrow). $\times 200$

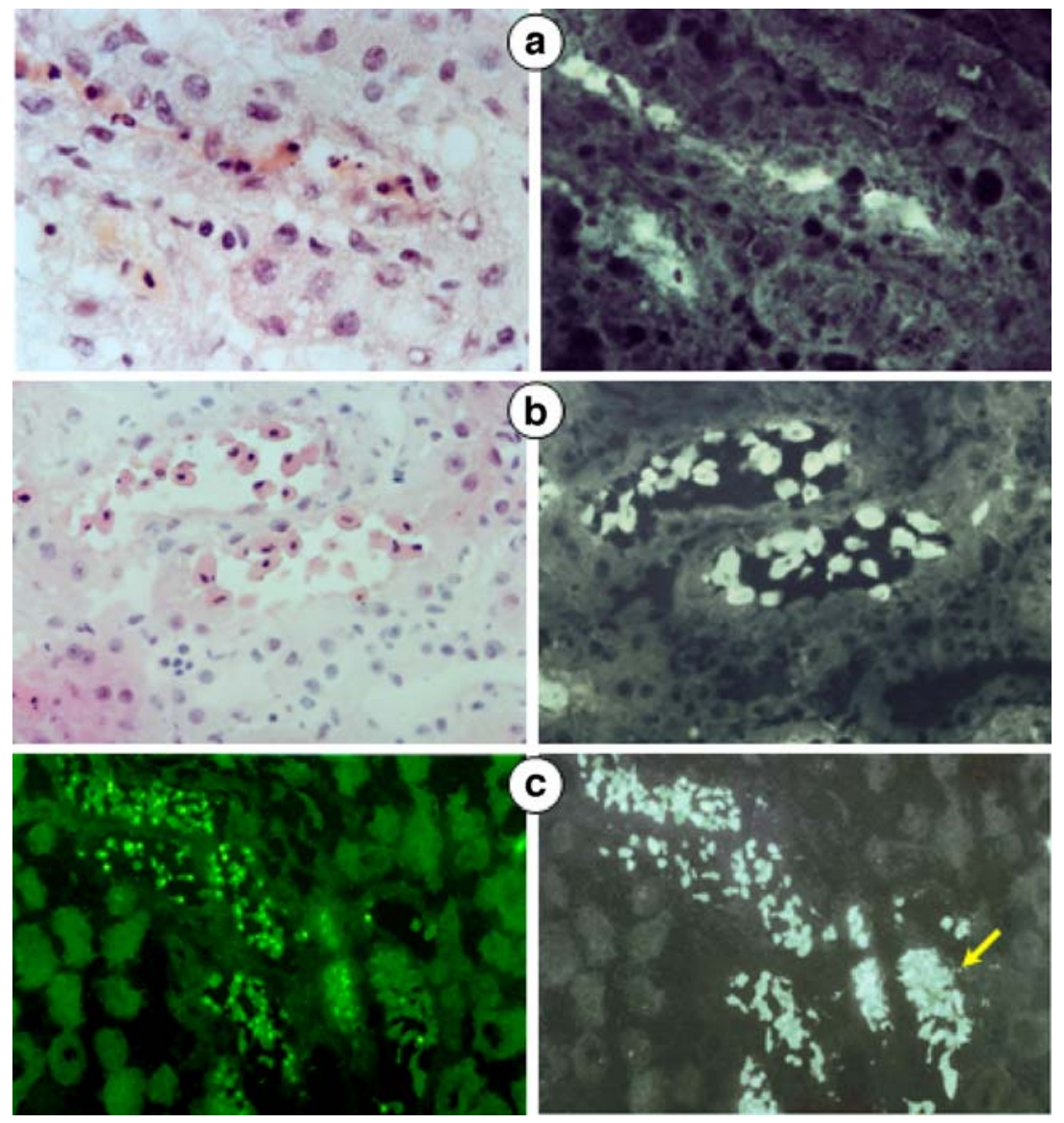

administered intraperitoneally. Renal ischemia was induced by unilateral left renal artery clamping, using a small nontraumatic vascular clamp, for $45 \mathrm{~min}$ [12]. Reperfusion was initiated by removal of the clamp. The period of renal reperfusion was $24 \mathrm{~h}$. Preliminary experiments revealed no difference between the I/R rat contralateral kidney and sham-operated normal rat kidneys with regard to cell death of renal tubular epithelium after $24 \mathrm{~h}$. Thus, in the present study, the contralateral, untreated kidney from the same animal served as the sham-operated control kidney. During the course of reperfusion, animals were injected intravenously with $20 \mathrm{mg} / \mathrm{kg}$ of DDC and $4 \mathrm{~h}$ later both kidneys were excised, frozen in liquid nitrogen, and stored at $-70^{\circ} \mathrm{C}$ until use. For microscopic analysis and fluorescence imaging, 5 - $\mu \mathrm{m}$-thick cryo-sections of both kidneys were evaluated either by fluorescence microscopy or were stained with hematoxylin and eosin (H\&E) for light microscopy analysis of apoptotic and/or necrotic lesion distribution. Furthermore, the presence of apoptotic cell nuclei was confirmed by apoptosis detection kit staining (ApopTag Fluorescein Kit, Intergen Company, Purchase, NY) using the TUNEL (terminal deoxynucleotidyl transferase-mediated uridine triphosphate nick end labeling) method. Additional ex vivo whole organ fluorescence imaging was carried out on freshly excised kidneys analyzed under a Leica (Leica MZ FL III, Leica, Switzerland) stereomicroscope. DDC was visualized using a $360_{\mathrm{Ex}}$ and $>420_{\mathrm{Em}} \mathrm{nm}(\mathrm{NU})$ filter set. Emitted fluorescence was collected on a Leica DC 300F digital camera and images were processed and analyzed by Leica QWin Lite software. For quantitative analysis of the extent of renal DDC accumulation, previously frozen kidneys were thawed, weighed, and homogenized in buffer containing $50 \mathrm{mM}$ Tris- $\mathrm{HCl}$ and $0.005 \%$ Triton X-100, pH 7.4 (1:7 w/v), using Heidolph RZR 2020 homogenizer (Heidolph Instruments GmbH \& Co. KG, Schwabach, Germany). Solubilized tissue extract samples were centrifuged twice at $13,800 \mathrm{rpm}\left(20 \mathrm{~min}, 4^{\circ} \mathrm{C}\right)$ and the supernatant fractions were analyzed using a microplate reader (GENious Fl Reader, TECAN,

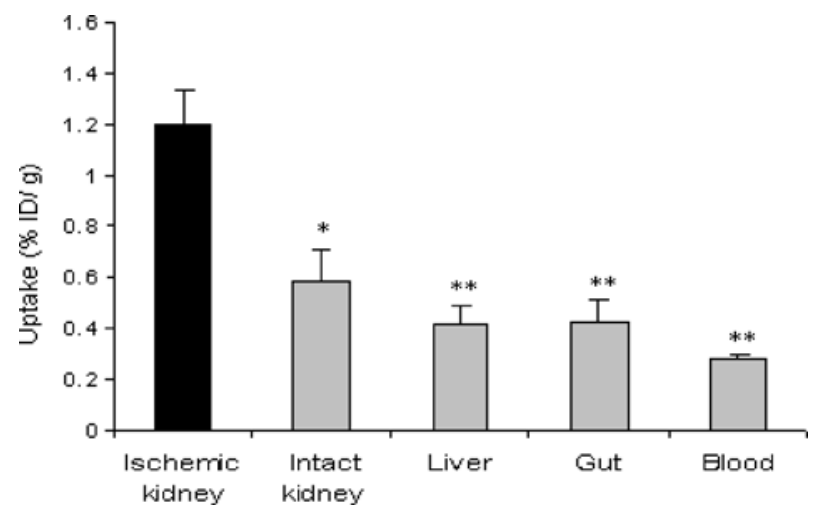

Fig. 5. Biodistribution of radiolabeled ${ }^{3} \mathrm{H}-\mathrm{DDC}$ after renal $\mathrm{I} / \mathrm{R}$ treatment. Uptake of ${ }^{3} \mathrm{H}-\mathrm{DDC}(\%$ injected dose per gram corrected for background) in target and non-target tissues, determined 18-24 h after ischemia and $4 \mathrm{~h}$ after ${ }^{3} \mathrm{H}-\mathrm{DDC}$ administration, is shown. Data are presented as mean \pm s.e.m., $n=7$. Asterisks denote significantly higher accumulation of ${ }^{3} \mathrm{H}-\mathrm{DDC}$ in ischemic kidney compared with other organs (tissues): ${ }^{*} p<0.05,{ }^{* *} p<0.01$ 
Grödig, Austria) equipped with optical filters for fluorescence measurements. All samples were pipetted in triplicate onto black Ritter flat bottom microplates and read at $360 \mathrm{~nm}$ Ex and $535 \mathrm{~nm}$ Em. For the calibration curve, a dilution series of DDC were prepared in the same Tris-Triton buffer, supplemented with $0.05 \%$ kidney extract of control vehicle-injected animals. The same control animal samples were also used for background values. In all samples, DDC concentration $(\mu \mathrm{g} / \mathrm{g}$ tissue) and the percentage of the injected dose $(\% \mathrm{ID} / \mathrm{g})$ were calculated.

Additional biodistribution studies were carried out to assess the spread of radiolabeled DDC into different organs. Twenty-four hours after unilateral renal ischemia/reperfusion, rats were intravenously injected with $5-20 \mu \mathrm{g} / \mathrm{kg}$ of the tritiated form of DDC $\left({ }^{3} \mathrm{H}-\mathrm{DDC}\right)$ [111-370 kBq (3-10 $\mu \mathrm{Ci}) /$ animal]. Four hours later, both kidneys, liver, gut, and blood samples were collected, weighed, and submitted to lyses by SOLVABLE reagent (GNE9100, Packard Bioscience), according to the manufacturer's instructions, and sample radioactivity was determined using a beta counter (TRI-CARB 2100TR, liquid scintillation analyzer, Packard Bioscience). Both tissue uptake (\% ID/ $\mathrm{g}$ ) and the ratios of the radioactivity of the ischemic kidney (DPM/g) to that of the contralateral kidney and the other non-target tissues were calculated.

\section{Rat model of radiocontrast-induced distal tubular necrosis} (DTN) The selectivity of DDC uptake in injured renal tissues was evaluated in this rat model, characterized by selective medullary hypoxic tubular damage. Nephropathy was induced by the combined administration of indomethacin (Sigma Chemical Co.), $10 \mathrm{mg} / \mathrm{kg}$, i.v., $\mathrm{N}$ - $\omega$-nitro-L-arginine methyl ester (L-NAME, Sigma Chemical Co.), $10 \mathrm{mg} / \mathrm{kg}$, i.v., and the radiocontrast agent sodium iothalamate $80 \%$ (Angio-Conray, Mallinckrodt Inc), $6 \mathrm{ml} / \mathrm{kg}$, i.a., as previously de- scribed [22]. The study was performed on six rats, with an additional two rats injected with vehicles serving as control (these two control animals studied are representative of at least 29 control rats tested in our previous experiments). Twenty-four hours after insult, animals were intravenously injected with $20 \mathrm{mg} / \mathrm{kg}$ DDC and $2 \mathrm{~h}$ later they were sacrificed. The left kidney was removed and snap-frozen for fluorescence analysis (and for H\&E and TUNEL staining, as detailed above), whereas the right kidney, used for morphological evaluation, was in vivo fixed with glutaraldehyde perfusion through the abdominal aorta, as described elsewhere [22, 23]. Perfusion-fixed kidneys were immersed in buffered $2 \% \mathrm{OsO}_{4}$, dehydrated, and embedded in an Araldite-EM bed 812 mixture. Large sections were cut perpendicular to the renal capsule, containing cortex and medulla, including fornix and papillae. One-micrometer sections were stained with methylene blue and analyzed in a blinded fashion (i.e., without knowing the fluorescence results) for morphological alterations. As previously detailed [23], tubular necrosis was determined separately for $\mathrm{S} 3$ proximal tubules in the outer stripe and medullary rays, and for medullary thick ascending limbs (mTALs) in the outer, mid, and inner zones of the inner stripe of the outer medulla. The extent of damage was expressed as the percentage of necrotic tubules out of total tubules counted. Papillary necrosis was semiquantitatively assessed using a score of $0-3$.

Sepsis model in the mouse The sensitivity of cell death recognition by DDC was tested in the cecal ligature and perforation (CLP) model in mice, where rare, focal tubular cell damage is hardly detected by regular morphological evaluation $[24,25]$. The cecum of six anesthetized mice was isolated, ligated distal to the ileocecal valve, and punctured twice with a 26-gauge needle. An additional four sham-treated animals were laparotomized, but the cecum was
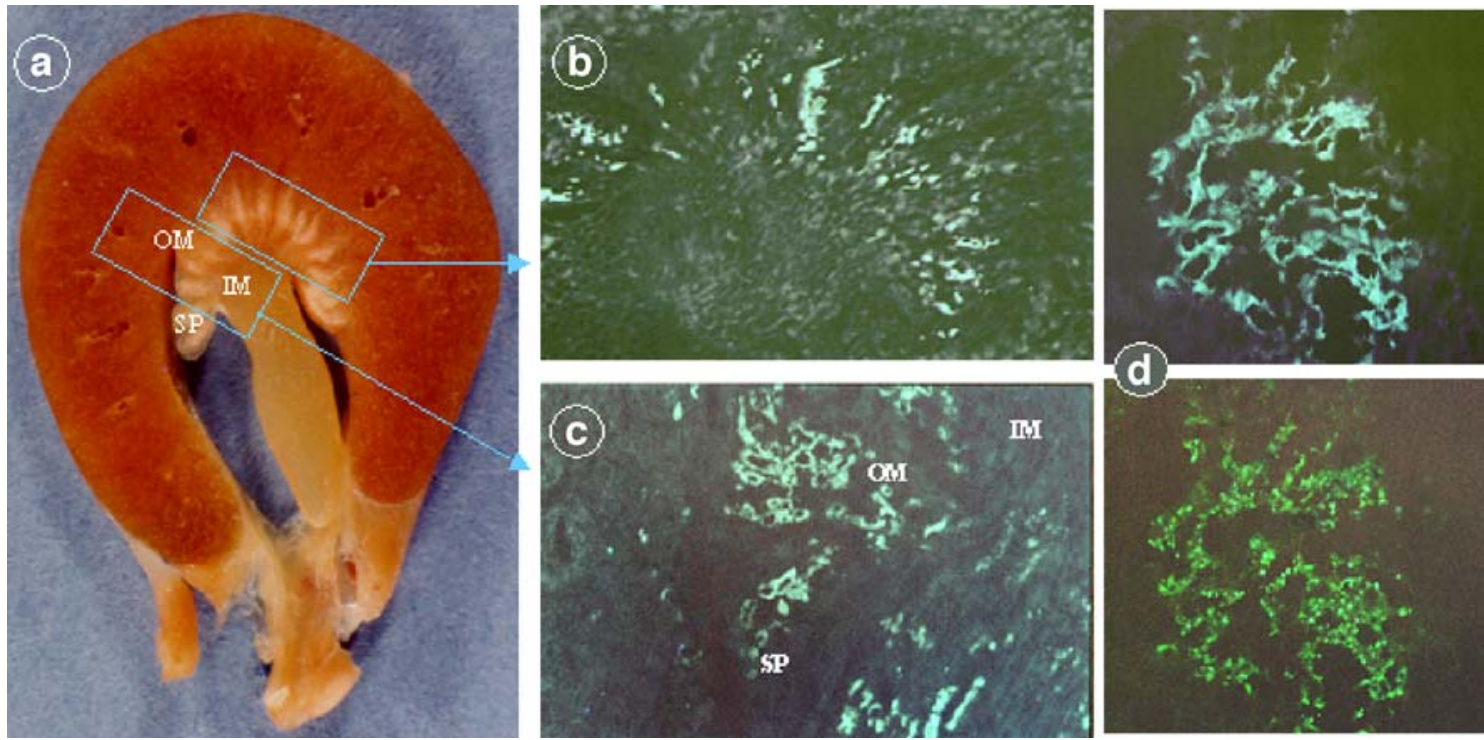

Fig. 6. Renal macroscopic and microscopic findings in rats subjected to the ARF protocol (indomethacin, L-NAME and iothalamate) and subsequently injected with DDC. a Macroscopic view of the characteristic striped pattern of severe hypoxic outer medullary damage in an in vivo fixed kidney. b The in vivo microscopic distribution of DDC in the contralateral kidney correlates closely with the distribution of the striped pattern of outer medullary necrosis in the ipsilateral one $(\times 30)$. c Details $(\times 100)$ of the cross-section, showing fluorescent imaging of DDC uptake in injured areas of both the inner strip and the forniceal region of the outer medulla. Examination of contiguous 5- $\mu \mathrm{m}$ sections revealed that the accumulation of DDC in damaged areas (d, upper panel) matched the infarct areas stained by TUNEL (d, lower panel) $(\times 200)$. OM outer medulla, $I M$ inner medulla, $S P$ secondary pyramid 
neither ligated nor punctured. Twenty-four hours later, mice were injected i.v. with DDC, and $2 \mathrm{~h}$ afterwards, kidneys were removed and subjected to fluorescence microscopy analysis as detailed above.

\section{Statistical analysis}

Data reported are mean \pm s.e.m. Student's $t$ test was used to assess the significance of differences between two groups. Significance was accepted at $p<0.05$

\section{Results}

\section{Binding of ApoSense to apoptotic cells in vitro}

Concomitant confocal imaging of HeLa cells undergoing apoptosis, double stained with DDC and annexin V, revealed that DDC accumulates within the cytoplasm of the apoptotic cell while annexin $\mathrm{V}$ is attached to the external apoptotic cell membrane (Fig. 1). Accumulation of ApoSense within apoptotic cells can contribute to ampli- fication of the signal obtained and consequently to the high signal-to-noise ratio. Previous studies have shown that through an interaction with the death receptor CD95 induces apoptosis by formation of a signaling complex at the cell membrane and subsequent caspase- 8 and caspase- 3 activation [26]. Therefore, the specificity of DDC uptake was tested in apoptotic Jurkat cells that were co-treated with caspase inhibitor and analyzed by flow cytometry. As shown in Fig. 2, in viable, non-apoptotic cells (green line), no uptake of DDC was detected, except in a small population of spontaneously dying cells representing late events. Following $150 \mathrm{~min}$ of incubation with anti-Fas, most cells accumulated DDC and created a new peak of cells (red line) with a higher fluorescence intensity (the geometric mean fluorescence intensity value, GMFI, was 120.18) while the peak of late apoptotic cells remained unchanged. In the presence of the caspase inhibitor ZVAD-FMK, cells lost the ability to bind DDC (black line), while their viability remained preserved, suggesting that accumulation of DDC compound is dependent on the apoptotic process itself. Upon addition of the caspase inhibitor, a marked shift towards a lower fluorescence
Fig. 7. Characteristic morphological findings in rats subjected to the ARF protocol and injected with DDC. Cryo-sections from the left kidney were used for DDC fluorescence analysis, whereas the in vivo fixed right kidney was used for light microscopy morphological examination of methylene blue-stained slides. Analyses of both kidneys were performed in a blinded fashion. An example is shown of the striped pattern reflecting the damage gradient, where tubules adjacent to the vasa recta $(V R)$ are preserved (red stars) while those distant from vasa recta are injured (black stars) (a, left panel, $\times 100)$. Note the excellent co-localization of morphologically damaged tubules with the DDC fluorescence $(\mathbf{a}$, right panel, $\times 100)$ in the contralateral kidney. DDC fluorescence was selectively identified in all morphologically injured regions, including papillary tip structures (b: left panel $\times 40$, right panel $\times 100$ )

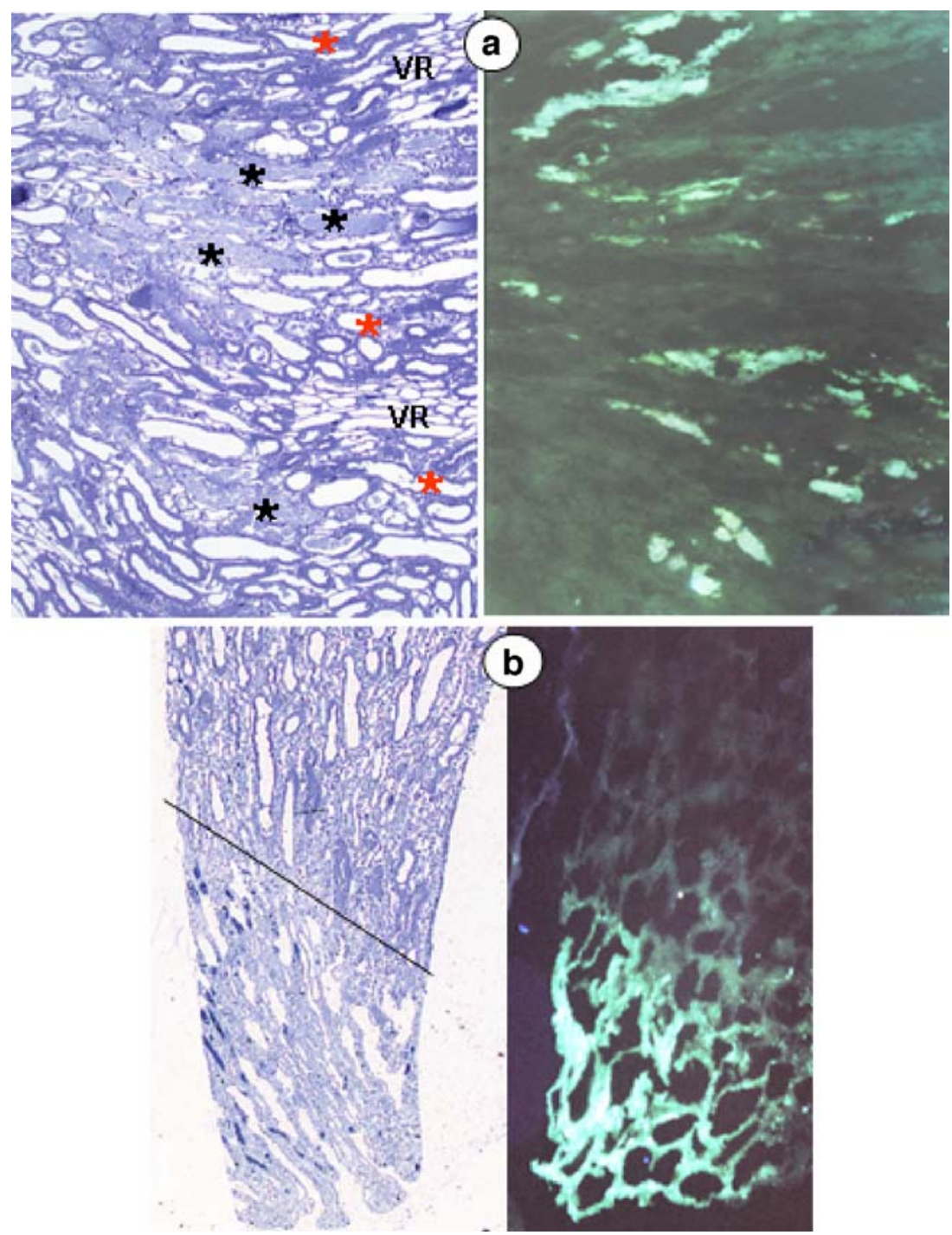


Fig. 8. Focal uptake of DDC in kidneys of six mice following sepsis caused by cecal ligature and perforation (CLP). Apoptotic cells, imaged by DDC, were shown either in the outer medulla (a, left) or in the cortex (a, right) of individual mice $(\times 100)$. Most apoptotic cells appeared to be tubular epithelial cells (b, left, arrows). An evident correlation was demonstrated between DDC fluorescence (b, right and $\mathbf{c}$, right) and apoptotic cells detected on $\mathrm{H} \& \mathrm{E}$ $(\mathbf{b}$, left $)$ or TUNEL (c, left $)$ stained slides $(\times 200)$
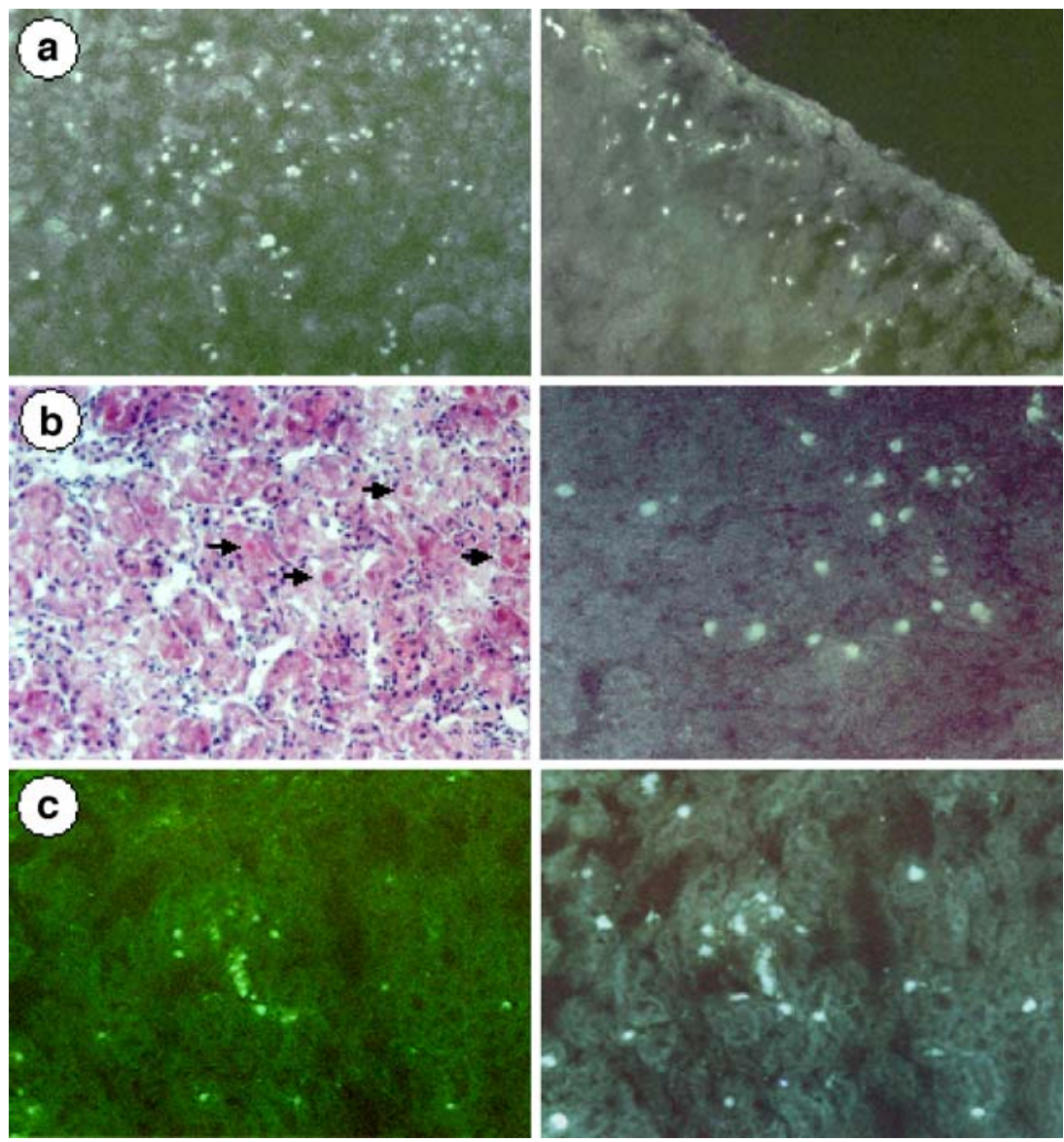

intensity was noted, and the final intensity values were almost the same as the control cell values (GMFI 46.38 and 46.02 , respectively), suggesting maximal inhibition.

\section{ApoSense uptake into ischemia/reperfusion (I/R) injured kidneys}

Evaluation of DDC accumulation into injured tubular cells of an ischemic rat kidney was performed on the basis of its intrinsic fluorescent properties. Either ex vivo whole kidney fluorescent imaging or cryo-section fluorescent analysis was employed. As seen in Fig. 3, DDC accumulated in the injured (a) but not in the contralateral intact kidney (b), thereby exhibiting substantial capability for imaging apoptosis and/or necrosis in vivo. Microscopic analysis of corresponding kidney sections revealed intense cytoplasmatic staining of tubular epithelial cells (Fig. 3c,d) located at the corticomedullary junction. No uptake of DDC into viable renal parenchyma was detected. The extent of DDC accumulation in these cells was quantitatively determined in homogenized whole kidney extracts from five rats, using microplate reader fluorescence measurement. As shown in Fig. 3e, the average DDC uptake into the ischemic kidney (expressed as $\% \mathrm{ID} / \mathrm{g}$ ) was $6.73 \pm$ 1.34 fold higher $(p=0.0076)$ than that in the intact control kidney $(0.41 \pm 0.11$ and $0.06 \pm 0.02$, respectively). H\&Estained histological sections of kidneys from I/R-treated rats revealed multiple apoptotic as well as necrotic cells situated along the tubule epithelium or in the tubule lumen (Fig. 4). A spectrum of changes characteristic of tubular cell apoptosis, including shrinkage of renal tubular cells, cytoplasmic acidophilia, chromatin dense condensation and margination, nuclear fragmentation, and pyknosis, is shown in Fig. 4a, left. In addition, as previously described [27-30], a large percentage of tubules in this region showed substantial tubular dilatation, epithelial necrosis, dissolving of tubular cells, luminal necrotic debris, and cast formation (Fig. 4b, left). The presence of apoptotic/necrotic cells was also defined by intensive green fluorescence of TUNEL-positive nuclei (Fig. 4c, left). As shown in Fig. $4 a-c$, right panel, the accumulation of DDC within the damaged areas in ischemic kidneys corresponds completely, even at the single cell level, to the injured areas detected by H\&E and TUNEL staining. However, it seems that in some areas, accumulation of DDC in apoptotic cell cytoplasm occurred earlier than DNA destruction and thereby cells were not stained by TUNEL but merely by DDC (Fig. 4c, arrow). These results may indicate that DDC has the ability to identify early apoptotic cells and that use of TUNEL alone may not reveal the full spectrum of cell death stages.

Biodistribution studies of ${ }^{3} \mathrm{H}-\mathrm{DDC}$ were performed in seven additional rats following the $\mathrm{I} / \mathrm{R}$ procedure. The accumulation of radioactivity in the blood, gut, liver and kidney at $4 \mathrm{~h}$ after intravenous administration is shown in 
Fig. 5. Uptake in the ischemic kidney was significantly higher than that in the intact contralateral one $(p<0.05)$ and also higher than liver, gut and blood uptake values $(p<0.01)$. The average uptake values at $4 \mathrm{~h}$, rendered as $\% \mathrm{ID} / \mathrm{g}$, were $1.20 \pm 0.13$ for the ischemic kidney, $0.58 \pm 0.12$ for the intact kidney, $0.41 \pm 0.07$ for the liver , $0.42 \pm 0.09$ for the gut, and $0.28 \pm 0.01$ for blood. The corresponding mean ischemic kidney-to-tissue ratios were $2.1 \pm 0.5,2.9 \pm$ $0.5,2.8 \pm 0.7$, and $4.3 \pm 0.6$, respectively. These results indicate that at $4 \mathrm{~h}$ following administration, DDC remained accumulated in the target organ, while being cleared from non-target ones.

\section{Radiocontrast-induced distal tubular necrosis}

Heyman et al. [23] reported previously that animals subjected to the radiocontrast model of DTN developed varying degrees of renal dysfunction and structural damage, but the presence, distribution, and extent of tubular injury on an individual basis could not be predicted from the magnitude of kidney dysfunction. In the present study, as well as in previously published studies [22, 23, $31]$, renal morphology analysis disclosed a wide-ranging extent of medullary (namely mTALs and S3 segments in the outer medulla and medullary rays) and papillary damage, with a poor overall correlation between structural damage and functional deterioration (data not shown). By contrast, as shown in Figs. 6 and 7, DDC distribution pattern and degree of deposition closely mirrored the morphological findings in the contralateral perfused kidney, suggesting that ApoSense uptake may reflect the level and degree of renal damage more closely than do functional tests. Results from both fluorescence and morphological analysis disclosed the characteristic striped pattern of injury (Fig. 6a,b), where tubules adjacent to the vasa recta are preserved while those distant from vascular bundles are injured (Fig. 7a). Homing of DDC was primarily restricted to injured regions within the outer medulla (Fig. 6c). No DDC uptake at a specified region in the absence of morphological damage was seen, as demonstrated in Fig. 7b, where necrosis of the papillary tip in one of the kidneys was clearly morphologically demarcated and DDC deposition was restricted to precisely the same injured area. Uptake occurred preferentially in apoptotic/necrotic lesions, as confirmed by co-localization with TUNEL staining (Fig. 6d). Thus, DDC was found to be a specific molecular marker for tubular injury in various segments of the kidney.

\section{Cecal ligature/perforation-induced sepsis}

DDC was evaluated as a potential in vivo marker for renal tubular cell apoptosis in another animal model of ATN, in which sepsis-associated renal dysfunction was induced by cecal ligation and puncture. Morphological alterations of the kidney due to sepsis can hardly be identified using routine histological techniques $[32,33]$, and experimental data regarding the apoptotic cells of kidneys affected by sepsis are therefore almost non-existent. Indeed, in all septic mice we found only rare focal renal tubular cell injury, seen either in the outer medulla or in the cortex (Fig. 8a). As seen in Fig. 8b, focal renal tubular cell injury is apparently identified by DDC fluorescence, but is not clearly traced using H\&E histopathological analysis; this demonstrates the high sensitivity of DDC in the detection of such renal damage. TUNEL staining confirmed the presumption that renal cells stained by DDC are indeed dying cells (Fig. 8c).

\section{Discussion}

As a result of recent progress in medicine, biology, and physics, the field of diagnostic imaging is shifting from conventional anatomical imaging to the sphere of functional and molecular imaging, with the aim of imaging biological processes related to both health and disease. The implementation of the new ApoSense technology for clinical imaging of apoptosis would represent a major addition to this emerging field, allowing non-invasive imaging of cell death processes in vivo as a means to both the diagnosis of disease and the monitoring of treatment efficacy in a broad spectrum of disease states.

In the present study, we have reported the successful application of ApoSense technology as a diagnostic imaging tool for the detection of apoptotic and/or necrotic cell death. ApoSense technology may be particularly useful in ARF, where current clinical and physiological assessment and complementary imaging tests are insufficient to discriminate between true renal parenchymal damage and prerenal azotemia (in which kidney filtration function is drastically reduced even though the internal structures of the kidney are intact). An inherent fluorescent property of one of the ApoSense family of compounds, DDC, was utilized for detection of tubular cell death in three animal models of ATN. The results obtained are in complete agreement with in vitro findings of DDC-specific binding to apoptotic cells (Figs. 1,2), and thereby strongly suggest the ability of DDC to image renal tubular cell apoptosis in vivo.

The three in vivo experimental models of ATN used in the present study complement each other regarding the applicability of ApoSense technology in the detection of ATN. The results obtained using the I/R model showed the qualitatively remarkable capability of ApoSense in targeting of injured tissues and ex vivo imaging of apoptosis (Fig. 3). Additionally, the model provides quantitative analysis of the extent of renal tubular cell damage based on the extent of DDC accumulation in these cells. It was shown that the uptake of DDC in the ischemic kidney was 6.73-fold higher than in the non-ischemic one. Moreover, the specificity of targeting of the injury by ApoSense was demonstrated by its localization to regions of apoptotic/ necrotic cell death, detected by H\&E and TUNEL staining (Fig. 4). Furthermore, it was observed that some TUNELnegative cells with a normal overall nuclear morphology 
were distinctively stained by ApoSense (Fig. 4c), conceivably representing cells in the initial phases of apoptosis. These results possibly indicate that ApoSense technology can provide a tool which will directly assess for early stages of programmed cell death in the ischemic kidney, before membrane vesicle formation and DNA degradation, particularly as measured by the TUNEL method [34].

The radiocontrast model of distal tubular necrosis provides a good example of specific targeting by ApoSense. Indeed, the presence of DDC was restricted to regions (medullary outer and inner stripe; papillary tip) that corresponded closely to the injured zones in the contralateral, perfusion-fixed kidney (Figs. 6, 7). Methylene blue-stained thin sections from those in vivo fixed kidneys enabled precise evaluation of the renal morphology and accurate identification of tubular cell types at various stages of evolving structural damage. The complete correlation between DDC fluorescence and histological evidence of damaged areas, mainly made up of apoptotic and necrotic cells, is indicative of the specificity of DDC uptake. Thus, while the degree of renal dysfunction served as an inaccurate indicator of tubular injury [4,5], ApoSense uptake closely predicted the existence of ATN, with an absolute specificity for involved tubular segments.

Our findings derived from the mouse sepsis model further illustrated the sensitivity of ApoSense in the detection of rare focal tubular cell injury that is not well defined by routine H\&E staining (Fig. 8). It has to be reiterated that data regarding the morphology of sepsisrelated ATN are nearly non-existent, mainly because such rare, focal tubular cell damage can hardly be detected by regular morphological evaluation $[35,36]$.

The inherent fluorescent properties of the ApoSense molecule DDC have been used in the present study to evaluate its specific binding to apoptotic cells. On the other hand, the structure of ApoSense molecules is modular, allowing for versatile attachment of various clinical imaging markers and, therefore, enabling their use as non-invasive imaging agents. In the current study, a ${ }^{3} \mathrm{H}-$ labeled DDC molecule was used for biodistribution and quantitative uptake studies. Nevertheless, autoradiography with radiolabeled DDC was not performed in this series of experiments and this may be the main limitation of the present study. Moreover, additional studies based on preparation of ApoSense as a PET imaging agent (following its conjugation with ${ }^{18} \mathrm{~F}$ ) are necessary before the introduction of the technology in the form of diagnostic imaging tests. These studies are currently in progress.

Overall, the results presented here are promising and illustrate the high sensitivity and specificity of ApoSense in the assessment of tubular cell damage in multiple experimental templates that may have clinically relevant applications. For instance, ApoSense technology may be implemented for the in vivo assessment of the degree to which renal parenchymal cell apoptosis is contributing to renal failure in sepsis and for the differentiation of evolving ATN from renal hemodynamic alterations; as a result it can enable therapy to be tailored to each patient's needs. The ability of radiolabeled derivatives of ApoSense to visualize both apoptosis and necrosis, non-invasively and in realtime, may prove useful in reducing the need for routine biopsy. Furthermore, in clinical practice, ApoSense imaging may provide diagnostic information superior to that obtained by means of biopsy, especially as regards the dynamic nature and focal distribution of the disorder; furthermore, it does not suffer from the risks and limitations associated with tissue sampling.

Imaging sciences have grown tremendously during recent decades, and many non-invasive techniques for the detection and monitoring of renal hemodynamics, such as advanced ultrasonography techniques, magnetic resonance imaging, and nuclear and X-ray computed tomography [37-39], have become essential clinical tools. Similarly, non-invasive technologies and imaging probes that would enable the detection of evolving renal parenchymal apoptotic and necrotic injury are critically needed. During the past few years, the best-characterized apoptosis marker, annexin V, a calcium-dependent phospholipid binding protein $[40,41]$, has been extensively investigated in multiple animal models as well as in clinical trials [42]. However, annexin $\mathrm{V}$ has a strong affinity for intact renal cortex, which has been attributed to the intrinsic lipid profile of the kidney, where a high concentration of phosphatidylserine exists [43, 44]. The high accumulation of annexin $\mathrm{V}$ in the kidneys precludes its use for the study of apoptosis in these organs or in their immediate vicinity $[45,46]$. In addition, the biological rationale for engineering DDC, a small non-peptide molecule with a molecular weight of $700 \mathrm{Da}$ (as compared with the $36-\mathrm{kDa}$ annexin $\mathrm{V}$ protein), is that such small molecules could benefit from less immunogenicity and the favorable biodistribution kinetics that small compounds in general exhibit over bigger proteins.

In summary, this study demonstrates that ApoSense, once successfully radiolabeled with an imaging moiety, may provide a sensitive means for real-time diagnosis and/ or monitoring of the extent of renal damage in ATN, utilizing selective targeting, binding, uptake, and accumulation within renal tubular apoptotic and/or necrotic cells. Moreover, as a non-invasive method, ApoSense imaging would allow longitudinal studies in a single individual, rendering important information on the optimal timing and dosing of drugs and on the efficacy of therapeutic interventions.

Acknowledgement. This research was performed and funded exclusively by NeuroSurvival Technologies (NST) Ltd.

\section{References}

1. Esson ML, Schrier RW. Diagnosis and treatment of acute tubular necrosis. Ann Intern Med 2002;137:744-52

2. Rosen S, Heyman SN. Difficulties in understanding human "acute tubular necrosis": limited data and flawed animal models. Kidney Int 2001;60:1220-4 
3. Heyman SN, Lieberthal W, Rogiers P, Bonventre JV. Animal models of acute tubular necrosis. Curr Opin Crit Care 2002;8:526-34

4. Racusen LC. Pathology of acute renal failure: structure/function correlation. Adv Renal Replace Ther 1977;42:3-16

5. Solez K, Morel-Maroger L, Sraer JD. The morphology of "acute tubular necrosis" in man; analysis of 57 renal biopsies and a comparison with the glycerol model. Medicine 1979;58:362-76

6. Sabbatini M, Sansone G, Uccello F, De Nicola L, Giliberti A, Sepe V, et al. Functional versus structural changes in the pathophysiology of acute ischemic renal failure in aging rats. Kidney Int 1994;45:1355-61

7. Liano F, Pascual J, Madrid Acute Renal Failure Study Group. Epidemiology of acute renal failure: a prospective, multicenter, community-based study. Kidney Int 1996;50:811-8

8. Molitoris BA. Acute renal failure. Drugs Today 1999;35:65966

9. Carvounis CP, Nisar S, Guro-Razuman S. Significance of the fractional excretion of urea in the differential diagnosis of acute renal failure. Kidney Int 2002;62:2223-9

10. Ichimura T, Bonventre JV, Bailly V, Wei H, Hession CA, Cate RL, et al. Kidney injury molecule-1 (KIM-1), a putative epithelial cell adhesion molecule containing a novel immunoglobulin domain, is up-regulated in renal cells after injury. J Biol Chem 1998;273:4135-42

11. Ichimura T, Hung CC, Yang SA, Stevens JL, Bonventre JV. Kidney injury molecule-1: a tissue and urinary biomarker for nephrotoxicant-induced renal injury. Am J Physiol Renal Physiol 2004;286:552-63

12. Lieberthal W, Levine JS. Mechanism of apoptosis and its potential role in renal tubular epithelial cell injury. Am J Physiol 1996;271:477-88

13. Rana A, Sathyanarayana P, Lieberthal W. Role of apoptosis of renal tubular cells in acute renal failure: therapeutic implications. Apoptosis 2001;6:83-102

14. Lieberthal W, Koh JS, Levine JS. Necrosis and apoptosis in acute renal failure. Semin Nephrol 1998;18:505-18

15. Beeri R, Symon Z, Brezis M, Ben-Sasson SA, Baehr PH, Rosen $\mathrm{S}$, et al. Rapid DNA fragmentation from hypoxia along the thick ascending limb of rat kidneys. Kidney Int 1995; 47:1806-10

16. Porter CJ, Ronan JE, Cassidy MJ. Fas-fas-ligand antigen expression and its relationship to increased apoptosis in acute renal transplant rejection. Transplantation 2000;69:1091-4

17. Hotchkiss RS, Swanson PE, Freeman BD, Tinsley KW, Cobb JP, Matuschak GM, et al. Apoptotic cell death in patients with sepsis, shock and multiple organ dysfunction. Crit Care Med 1999;27:1230-51

18. Oberholzer C, Oberholzer A, Clare-Salzler M, Moldawer LL. Apoptosis in sepsis: a new target for therapeutic exploration. FASEB J 2001;15:879-92

19. Lieberthal W, Nigam SK. Acute renal failure. II. Experimental models of acute renal failure: imperfect but indispensable. Am J Physiol Renal Physiol 2000;278:F1-12

20. Wu ZL, Ge YQ. An animal model of septicemia-induced hypercatabolic acute renal failure. Ren Fail 1999;21:477-82

21. Weisberg LS, Allgren RL, Genter FC, Kurnik BR, The Auriculin Anaritide Acute Renal Failure Study Group. Cause of acute tubular necrosis affects its prognosis. Arch Intern Med 1997; 157:1833-8

22. AgmonY, Peleg H, Greenfeld Z, Rosen S, Brezis M. Nitric oxide and prostanoids protect the renal outer medulla from radiocontrast toxicity in the rat. J Clin Invest 1994;94:1069-75
23. Heyman SN, Fuchs S, Jaffe R, Shina A, Ellezian L, Brezis M, et al. Renal microcirculation and tissue damage during acute ureteral obstruction in the rat: effect of saline infusion, indomethacin and radiocontrast. Kidney Int 1977;51:653-63

24. Baker C, Chaudry IH, Gains HO, Baue AE. Evaluation of factors affecting mortality rate after sepsis in a murine cecal ligation and puncture model. Surgery 1983;94:331-5

25. Remick DG, Newcomb DE, Bolgos GL, Call DR. Comparison of the mortality and inflammatory response of two models of sepsis: lipopolysaccharide versus cecal ligation and puncture. Shock 2000;13:110-6

26. Hirata H, Takahashi A, Kobayashi S, Yonehara S, Sawai H, Okazaki T, et al. Caspases are activated in a branched protease cascade and control distinct downstream processes in Fasinduced apoptosis. J Exp Med 1998;187:587-600

27. Ueda N, Shah SV. Tubular cell damage in acute renal failureapoptosis, necrosis, or both. Nephrol Dial Transplant 2000;15: 318-23

28. Lieberthal W, Fuhro R, Andry CC, Rennke H, Abernathy VE, Koh JS, et al. Rapamycin impairs recovery from acute renal failure: role of cell-cycle arrest and apoptosis of tubular cells. Am J Physiol Renal Physiol 2001;281:F693-706

29. Padanilam BJ. Cell death induced by acute renal injury: a perspective on the contributions of apoptosis and necrosis. Am J Physiol Renal Physiol 2003;284:F608-27

30. Tsuruya K, Tokumoto M, Ninomiya T, Hirakawa M, Masutani $\mathrm{K}$, Taniguchi $\mathrm{M}$, et al. Antioxidant ameliorates cisplatininduced renal tubular cell death through inhibition of death receptor-mediated pathways. Am J Physiol Renal Physiol 2003;285:F208-18

31. Heyman SN, Goldfarb M, Shina A, Karmeli F, Rosen S. $N$ acetylcysteine ameliorates renal microcirculation: studies in rats. Kidney Int 2003;63:634-41

32. Cunningham PN, Dyanov HM, Park P, Wang J, Newell KA, Quigg RJ. Acute renal failure in endotoxemia is caused by TNF acting directly on TNF receptor-1 in kidney. J Immunol 2002;168:5817-23

33. Morikawa A, Sugiyama T, Kato Y, Koide N, Jiang GZ, Takahashi K, et al. Apoptotic cell death in the response of Dgalactosamine-sensitized mice to lipopolysaccharide as an experimental endotoxic shock model. Infect Immun 1996; 64:734-8

34. Martin SJ, Reutelingsperger CP, McGahon AJ, Rader JA, van Schie RC, LaFace DM, et al. Early redistribution of plasma membrane phosphatidylserine is a general feature of apoptosis regardless of the initiating stimulus: inhibition by overexpression of Bcl-2 and Abl. J Exp Med 1995;182:1545-56

35. Miyaji T, Hu X, Yuen PS, Muramatsu Y, Iyer S, Hewitt SM, et al. Ethyl pyruvate decreases sepsis-induced acute renal failure and multiple organ damage in aged mice. Kidney Int 2003; 64:1620-31

36. Holly MK, Dear JW, Hu X, Yuen PST, Yasuda H, Iyer SN, et al. Clinically relevant rat model for sepsis-induced acute kidney disease [abstract]. J Am Soc Nephrol 2004;15:457A

37. Heuer R, Sommer G, Shortliffe LD. Evaluation of renal growth by magnetic resonance imaging and computerized tomography volumes. J Urol 2003;170:1659-63

38. Kobayashi H, Jo SK, Kawamoto S, Yasuda H, Hu X, Knopp MV, et al. Polyamine dendrimer-based MRI contrast agents for functional kidney imaging to diagnose acute renal failure. J Magn Reson Imaging 2004;20:512-8

39. Noble VE, Brown DF. Renal ultrasound. Emerg Med Clin North Am 2004;22:641-59 
40. Koopman G, Reutelingsperger CP, Kuijten GA, Keehnen RM, Pals ST, van Oers MH. Annexin V for flow cytometric detection of phosphatidylserine expression on B cells undergoing apoptosis. Blood 1994;84:1415-20

41. Tait JF, Gibson D. Measurement of membrane phospholipid asymmetry in normal and sickle-cell erythrocytes by means of annexin V binding. J Lab Clin Med 1994;123:741-8

42. Lahorte CM, van de Wiele C, Bacher K, van den Bossche B, Thierens $\mathrm{H}$, van Belle $\mathrm{S}$, et al. Biodistribution and dosimetry study of ${ }^{123} \mathrm{I}$-rh-annexin $\mathrm{V}$ in mice and humans. Nucl Med Commun 2003;24:871-80

43. Blankenberg FG, Katsikis PD, Tait JF, Davis RE, Naumovski $\mathrm{L}$, Ohtsuki $\mathrm{K}$, et al. In vivo detection and imaging of phosphatidylserine expression during programmed cell death. Proc Natl Acad Sci U S A 1998;95:6349-54
44. Sterin-Speziale N, Kahane VL, Setton CP, Fernandez MC, Speziale EH. Compartmental study of rat renal phospholipid metabolism. Lipids 1992;27:10-4

45. Kemerink GJ, Liem IH, Hofstra L, Boersma HH, Buijs WC, Reutelingsperger $\mathrm{CP}$, et al. Patient dosimetry of intravenously administered ${ }^{99 \mathrm{~m}}$ Tc-annexin V. J Nucl Med 2001;42:382-7

46. Boersma HH, Liem IH, Kemerink GJ, Thimister PW, Hofstra L, Stolk LM, et al. Comparison between human pharmacokinetics and imaging properties of two conjugation methods for ${ }^{99 \mathrm{~m}}$ Tc-annexin A5. Br J Radiol 2003;76:553-60 